\title{
User Acceptance Evaluation of a Gamified Knowledge Sharing Platform for Use in Industrial Environments
}

\author{
Stylianos Zikos ${ }^{1}$, Maria Tsourma ${ }^{1}$, Evdoxia E. Lithoxoidou ${ }^{1}$, Anastasios Drosou ${ }^{1}$, \\ Dimosthenis Ioannidis ${ }^{1}$, Dimitrios Tzovaras ${ }^{1}$ \\ ${ }^{1}$ Information Technologies Institute, Centre for Research and Technology Hellas, \\ Thessaloniki, Greece \\ \{szikos, mtsourma, elithoxo, drosou, djoannid,Dimitrios.Tzovaras\}@iti.gr
}

\begin{abstract}
This study evaluates user acceptance of a gamification-enabled collaboration and knowledge sharing platform that has been developed for use by personnel in industrial work environments, aiming at increasing motivation for knowledge exchange. The platform has been evaluated at two manufacturing industries by two groups of users, workers and supervisors, with regard to five criteria: usability, knowledge integration, working experience, user acceptance and overall impact. Results showed that even though the ratings from both industries were positive on all criteria, there is room for improvement on user acceptance and knowledge integration. Driven by this fact, a rule-based adaptive gamification approach which exploits information about workers is proposed in order to further increase motivation and engagement. Based on feedback received from the evaluation, guidelines related to functionalities and design of a gamified collaboration platform are provided. These guidelines can be followed when implementing collaboration tools with gamification support for industrial environments.
\end{abstract}

Keywords: gamification, collaboration platform, behavior monitoring;

\section{Introduction}

Over the past years, large organizations have adopted social networking platforms to improve collaborative communication, however, most of them have experienced little or no success [1]. This is due to the fact that successful use of such kind of platforms is often linked to positive organizational culture, which are the behaviours that contribute to the unique social and psychological environment of an organization. In a changing environment the culture has to evolve as well. In [2], six guidelines for culture change are proposed, which are the following: (i) Formulate a clear strategic vision, (ii), display top-management commitment, (iii) model culture change at the highest level, (iv) modify the organization to support organizational change, (v) socialize newcomers and terminate deviants and (vi) develop ethical and legal sensitivity. Encouraging employee motivation and employee training to new processes and systems, are important steps towards a healthy culture.

Collaboration and knowledge sharing platforms must have certain characteristics in order to be successful, especially when used in industrial work environments, such as to be simple to use, and motivate engagement of workers for both requesting assistance and sharing their expertise to colleagues. The latter can be realized through the application of the gamification concept. Gamification - the use of game design elements in non-game contexts - has seen rapid adoption in recent years. However, there are challenges and open research questions in the design of gameful systems that need to be addressed, such as the 
organization of design teams, and whether it is preferable to design a system from scratch or extend an existing one, and under which conditions [3]. According to a broader definition, a serious game is any piece of software that merges a non-entertaining purpose with a video game structure [4]. The gamification concept in industrial work environments is a recent trend and can be applied to different cases, such as for making the execution of repetitive tasks more interesting and joyful, motivating workers to perform recommended tasks that often neglect, and motivating workers to share knowledge, exchange ideas and view training content (e.g. videos). The present paper focuses on the last case.

User acceptance evaluation events gather input from actual system users to determine where potential problems may exist in new software or major upgrade [5]. User acceptance evaluations of software solutions that are designed to be used by employees in working environments are important, as useful conclusions can be reached about the usability and fulfillment of the design goals. A poorly designed software application can discourage users from using it. Furthermore, a solution which is intended to be used in a work environment must satisfy both workers and stakeholders, meeting the objectives of all types of users. The feedback acquired from such evaluations is valuable as it can be used for improving the provided functionalities or adapting them to the organization's specific needs.

This paper presents a social collaboration platform that has been developed for use in industrial work environments. The platform makes use of a gamification engine and provides the necessary user interface elements in order to increase user motivation. The main advantage of the gamification engine presented in this work is that it allows to create actions and rules that can be triggered by external systems utilized in modern industries, such as a training software application. The contributions of this paper are the following:

a. User evaluation results are presented and discussed after the installation and use of the web-based social collaboration platform with gamified tasks at two industries.

b. Guidelines regarding functionalities and design of a gamified collaboration platform are provided, based on feedback received from users.

c. Novel gamification scenarios that exploit additional information available in modern factories are proposed, which can be applied in order to further improve users' motivation and engagement.

The paper is organized as follows: Section 2 presents related work on the application and evaluation of gamification in industrial environments. Section 3 describes the features and architecture of the gamification-enabled collaboration platform. User acceptance evaluation results are presented and discussed in Section 4. Section 5 presents an adaptive gamification concept that can be applied towards increasing the impact of gamification on the use of the collaboration platform. Lastly, the conclusions are summarized in Section 6.

\section{Related work}

According to Hamari et al. [6], a large number of studies have been conducted in recent years presenting gamification applications in a wide range of contexts, such as education [7-9], work [10-12], and innovation [13], among others. Education and knowledge sharing are two fields that have high potential to engage gamification as they seek to strengthen motivation and engagement [14]. In [15], Petridis et al. provide a review of existing literature on the use of serious games in the business environment. Their findings indicate that serious games can have positive impacts in training, decision-support, and consumer outreach. They also focus on the challenges and pitfalls when applying gamification principles within a business context. The interaction and linking between the development of a serious game and the game's business model is explored in study [16] by Moller and Hansen. Their research indicates that the business model behind the game can have a significant effect on the final version(s) of the game. The authors of [17] examine the use of gamification in an IoT-enabled mobile application that provides personalized recommendation tips to employees for adopting a more green behavior at the workplace. 
Questionnaires results revealed that the application has potential to be used if includes at least three game design elements: progression, levels and points. In paper [18], Jurado et al. explore an approach which incorporates gamification mechanics to improve participation in knowledge management processes. Results showed that gamification improved aspects like participation, contribution and knowledge refinement in the process of software development. In [19] the authors study the increasing adoption of enterprise social networking platforms by organizations, and present survey results from business professionals to examine current views of these platforms.

User acceptance evaluations are commonly used in the literature for studying the impact of new methods and software tools on users. For example, the authors of [20] investigate how user interface design affects older people's intention and attitude related to using social networking sites. To this end, they evaluate a social application specifically designed for the elderly. User acceptance evaluations of gamified processes utilized in software platforms are also found in the literature. For example, the acceptance of gamified work processes in the automotive industry is studied by Korn et al. in [21]. Results revealed that the general acceptance was high when gamification is adapted to the requirements of the production domain. In paper [22], Witt et al. study the effect of game mechanics, such as points or leaderboards, within an online idea competition using participants' feedback through questionnaires. The results showed that if game mechanics are applied inadequately, their efficiency is not high.

Adaptive gamification [23] is a new concept which enhances traditional gamification approaches with the use of user-centered incentive mechanisms, for creating a personalized gamification experience. A literature review about current developments and challenges in adaptive gamification can be found in [24], where the authors also highlight five research challenges. Understanding the relationship between the mechanics and their effects on different users, is one of these challenges. A design framework for systematic development of adaptive gamification applications is proposed by Böckle et al. in [25]. In that work, the evaluation in an online knowledge exchange platform indicated positive user acceptance and increased usage of the system. The authors of study [26] explore the adaptive gamification approach for learning environments. In particular, they present an adaptive gamification model based on a linear model between player types and gamification features. The implemented system selects gaming features by considering the player type of the user and results showed that learners with adapted gaming features spend more time in the learning environment.

The benefits that can be derived from the user acceptance evaluation study of this paper are the following: (a) It is possible to investigate whether context of use can significantly affect user acceptance, as evaluation of the gamified collaboration platform is performed at two different industries. (b) Participation of two distinct types of users who have different roles (workers, supervisors), allows to evaluate all aspects of the system, i.e. participation in gamified tasks as well as the management of the implemented gamification engine. Lastly, the system described in this paper allows factory managers to examine whether a Standard Operating Procedure is acceptable from the workers or the supervisors when they provide remote support. This is a unique approach that is fully parameterized through the provisioned gamification engine.

\section{System description}

The conceptual view of the system, which is a gamification-enabled collaboration platform, is illustrated in Figure 1. The system is composed of a web-based collaboration platform and a gamification engine. These components interact with each other, as the former provides to the gamification engine the actions triggered, and retrieves status information which is visualized in the user interface. The collaboration platform and the gamification 
engine store data in their own database, so that exploitation of information stored can be done easily, and independently of any other integrated tool.

The main objective of the collaboration platform is to enhance social interaction among workers, save time and provide the means for sharing knowledge towards problem solving and satisfaction increase. The goal of the gamification engine is to support game mechanics in various user activities on the collaboration platform, in order to motivate workers to use the system, upload content and share their knowledge and work experiences to co-workers. Moreover, gamification engine aims at empowering activities that are of no interest and at enhancing an environment with playful elements in order to extend engagement.

The collaboration platform provides to workers a place to (a) exchange knowledge with their colleagues through discussions, (b) request support for resolving high priority incidents, and (c) view and share multimedia content related to tasks and use of equipment. For the implementation of the aforementioned functionalities, various social media technologies have been included, such as discussion boards, multimedia-based news feed, and instant messaging. Furthermore, to improve social interaction among colleagues, groups that allow sharing of activities can be created. Implementation of the collaboration platform is based on previous work described in [27].

Certain actions of the collaboration platform have been gamified with the use of the gamification engine. Users of the platform can collect points when performing actions that are useful for the community or actions related to skills enrichment (e.g. watching training videos). The gamification engine provides API calls to web services through which any tool can be connected to the engine and gamify its actions. Therefore, it can also be used to gamify tasks that are not related to the use of the collaboration platform. Team-based gamified tasks, supported by the gamification engine, are ideal for an industrial work environment, however, competition among different teams should not be encouraged. For example, a game to promote collaboration among workers can be created using the gamification engine, involving a recommended but yet important task that is often neglected at the shop floor. In this game, only one team of workers participate (e.g. employees working in a given shift), which competes to its own result of the previous day. As a result of this approach, there will be no workers with the negative feeling of losing the game.

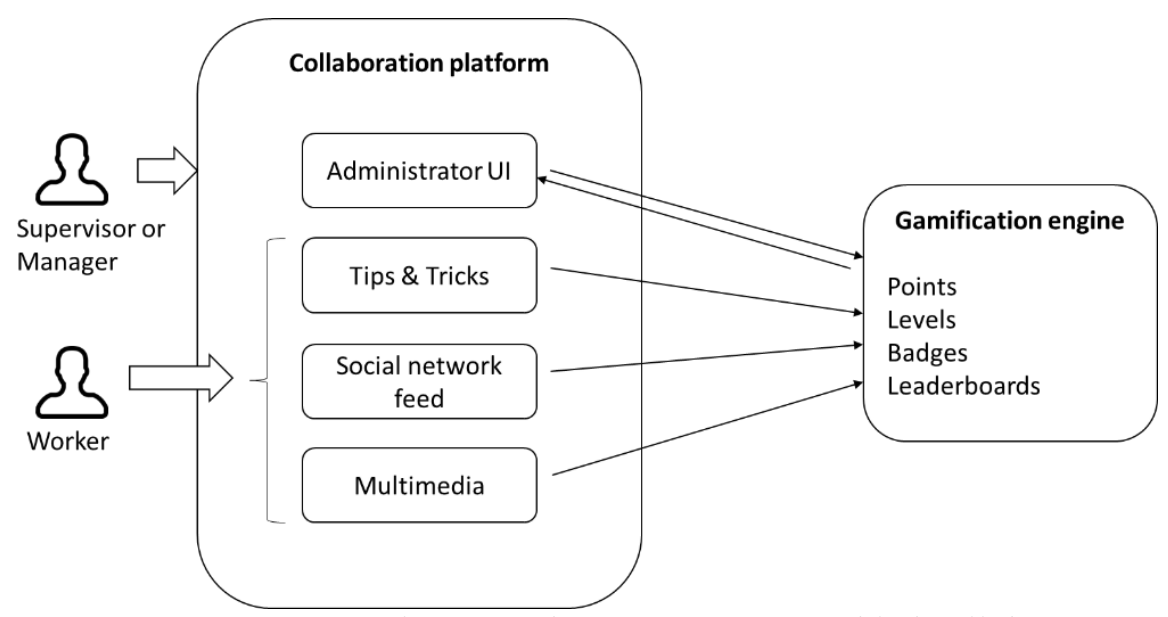

Figure 1. Conceptual view of the gamification-enabled collaboration platform

Two workflows with regard to the integration of gamification into the collaboration platform are supported by the system: Management of gamified tasks, and user participation in gamified tasks. Gamified tasks management refers to the creation, modification, and deletion of gamified tasks by the administrator. A supervisor or manager of the industry can be the administrator of the platform. Configuration of gamification engine by an end user is a main feature, as it offers convenient adaptation to changing needs. Figure 2 shows 
the steps required for creating a new gamified task by the administrator via the user interface. At first, the game category has to be selected. It can be either individual or team based. In the latter case, all users belonging to the same team contribute to the collection of points and awards. Optionally, the administrator can set possible levels that can be reached, and define the required points for each level. If this is not the case, all participants are by default on Level 1, which is the automatically created base level so as to motivate implementation of additional levels. Subsequently, supported actions are added. In this particular implementation, actions refer to user actions that can be made through the interface of the collaboration platform. The awards of the game are defined next. Different types of awards are supported, such as scalar, set, and tangible awards. Scalar awards are earned based on collected points, while set awards are earned based on achievements. The administrator can combine levels, actions, and awards by using the rule engine. Rules are fundamental for a gamified procedure as they define the behavior that a user must follow in order to achieve the award. Different types of rules are considered, such as action-based rules, awards-based rules, and rules used for advancing to the next level. A multilingual interface for the end users is available, as the gamification engine offers the ability to select display language during the game creation process.

A user is able to view the available games and select the ones to join based on a brief description as well as a popularity metric that shows the percentage of the registered participants that have already joined. Once joined to a game, his/her gamified actions can be triggered and are passed as input to the rule engine. The user can leave a game at any time. Workflow when participating in a gamified task is shown in Figure 3. A tracking mechanism is implemented to the platform in order to log actions and keep historical data for extracting useful information about how the platform is being used. Consequently, games or specific actions that need refinements can be discovered. Each gamified action, triggered by the user, is logged along with the timestamp, the ID of the user, current score, and the ID of the game. Furthermore, a sequence of activities performed by the administrator of the platform are recorded as well in order to keep historical data for analysis. Activities like adding or deleting a game, or changing rules in a game can reveal success or failure in gamification design. Additionally, joining or leaving games are also logged as these actions show engagement to the corresponding activities. Thus, the analysis of these logs helps improving rules for keeping strong motivation.

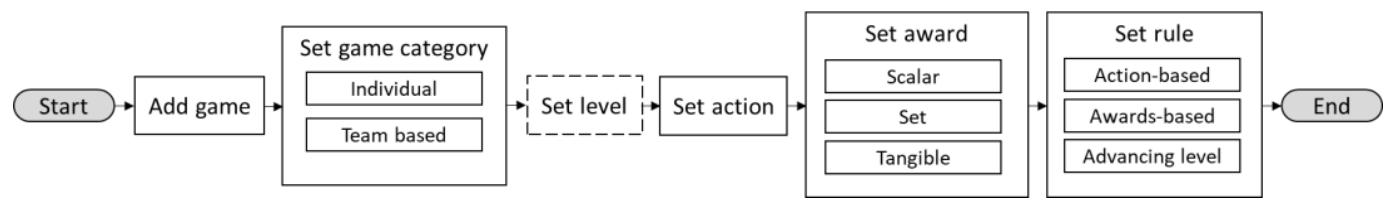

Figure 2. Gamified task creation process 


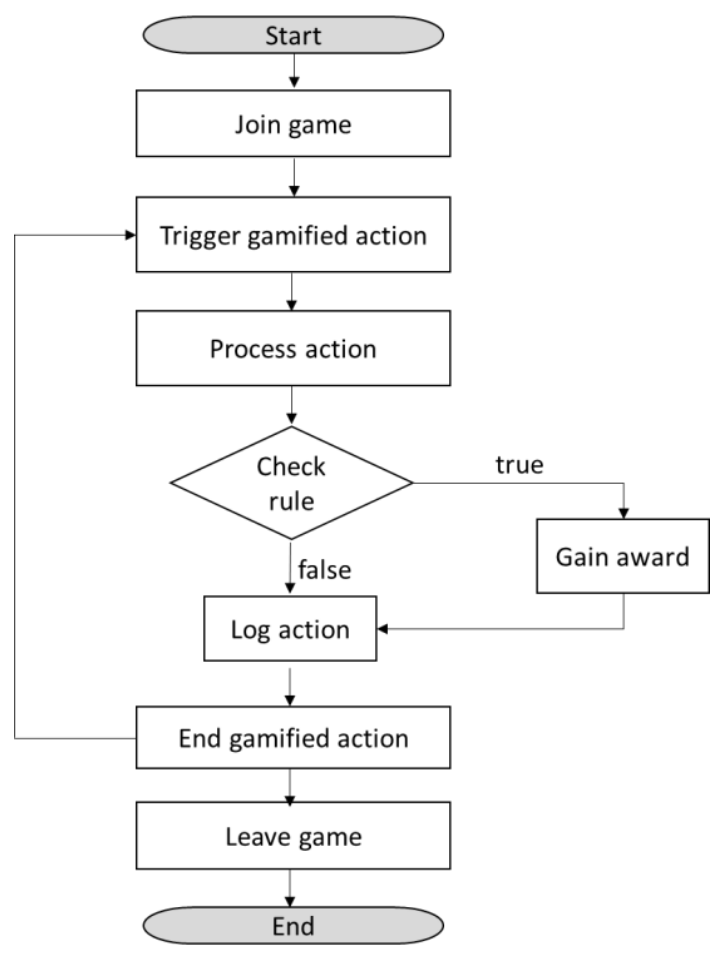

Figure 3. Workflow when participating in a gamified task

A game which includes multiple gamified actions, applied through the collaboration platform, has been created for evaluation in real production environments. The objective of this game is to promote the use of the collaboration platform. The list of gamified actions included is shown in Table 1. According to the gamified actions defined, users gain points when they make a question or answer a question in the forum (Figure 4), upvote an answer of another user, or watch multimedia content for training. Awards in the form of badges were also defined. Cup badges (bronze, silver, golden) were available based on the total number of collected points. Moreover, a special badge is acquired if the user has watched the full set of training videos ( 10 videos). Figure 5 presents the user interface provided to the administrator when creating a game. Fields to enter generic game information and fields for the definition of supported gamification elements are available to the administrator. Firstly, the administrator enters the generic attributes of a game such as name and type. Then, administrator defines the actions, quests, awards, rules, and levels of the game. Figure 6 shows the interface for creating a new award, where the administrator sets its name, description, type and attributes. Figure 7 presents the interface provided to the worker through the 'My profile' tab, where information about the games is presented. In particular, the worker is able to view the list of games, where current level, number of points collected, popularity, and awards are shown per each game.

Table 1. List of gamified actions

\begin{tabular}{c|l}
\hline Action & \multicolumn{1}{c}{ Points are awarded to user when... } \\
\hline Training & ...participating in a training session \\
\hline Ask question & ... asking a question in the forum \\
\hline Post answer & ... answering a question \\
\hline Upvote answer & ... upvoting an answer in the forum \\
\hline
\end{tabular}




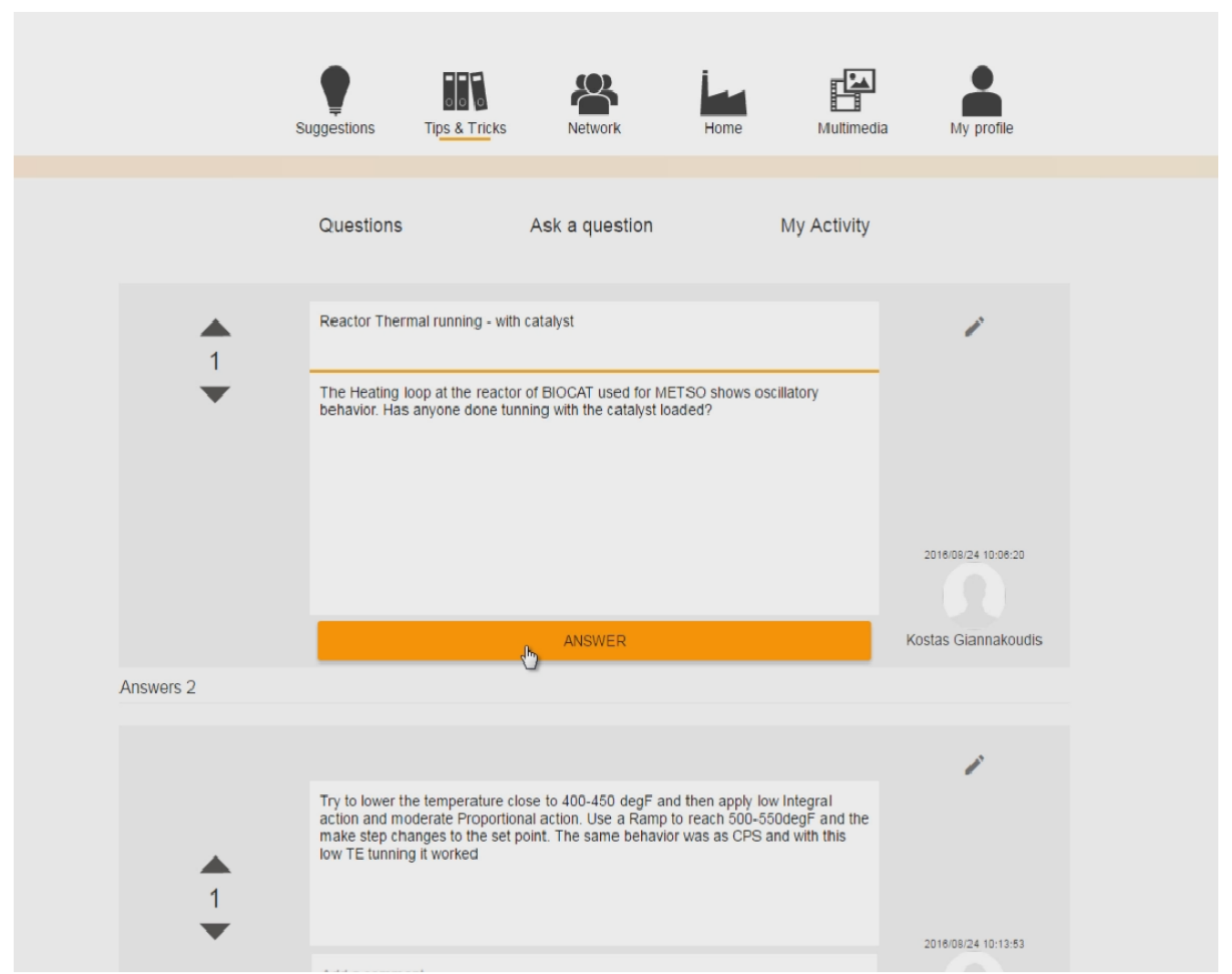

Figure 4. Discussion forum where users ask and answer questions

Admin Home Network Multimedia Tips \& Tricks Suggestions My profile | Admin Admi... R A If |

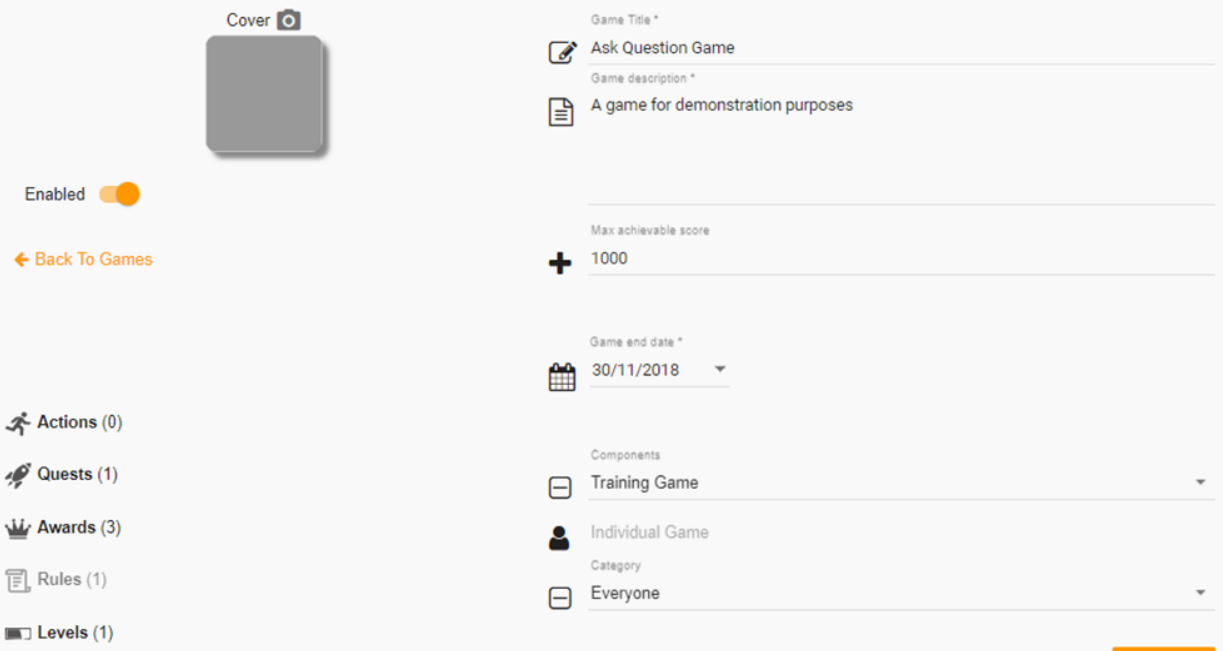

Figure 5. Administrator's interface for creating a gamified task (game). Generic attributes of the game and elements shown on the left, are defined 
pag. 96

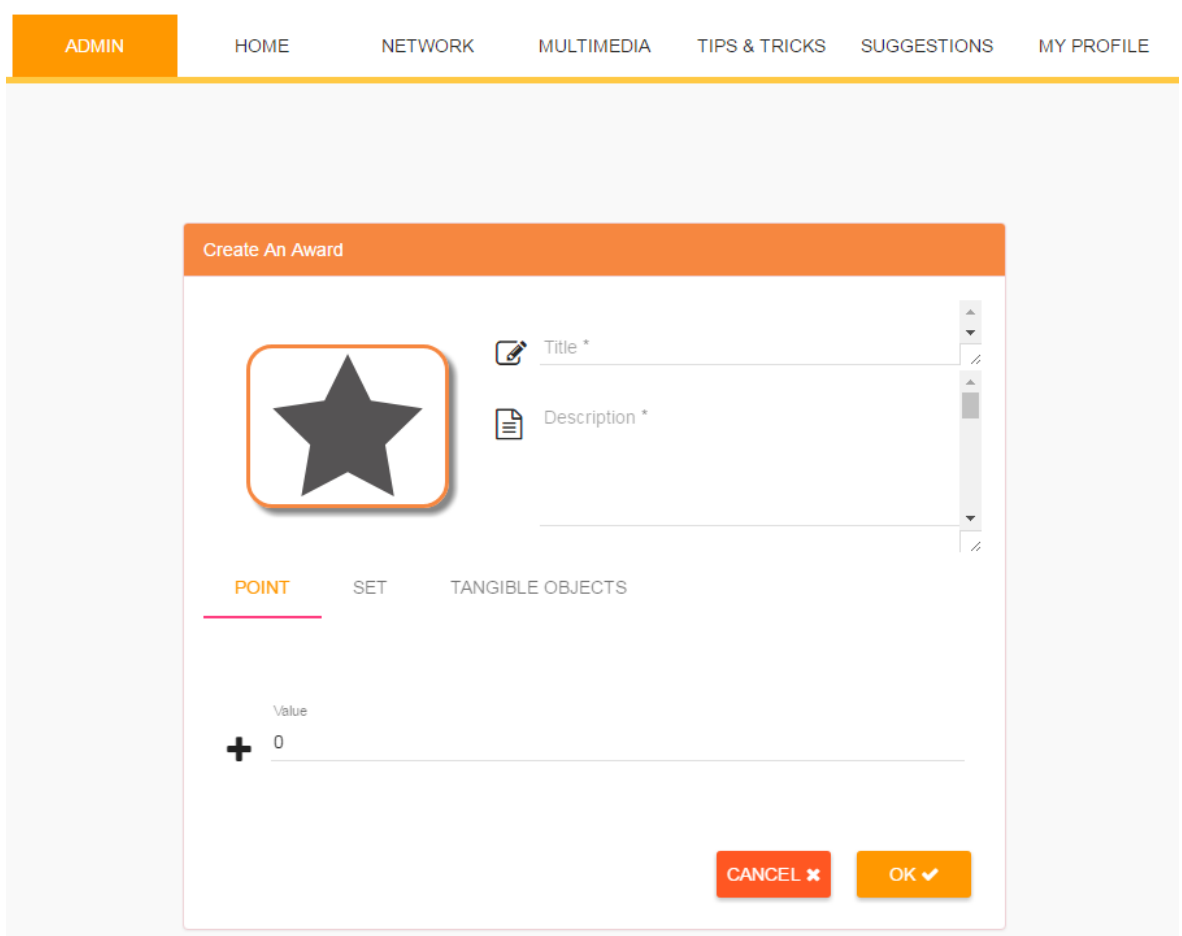

Figure 6. Administrator's interface for creating a new award

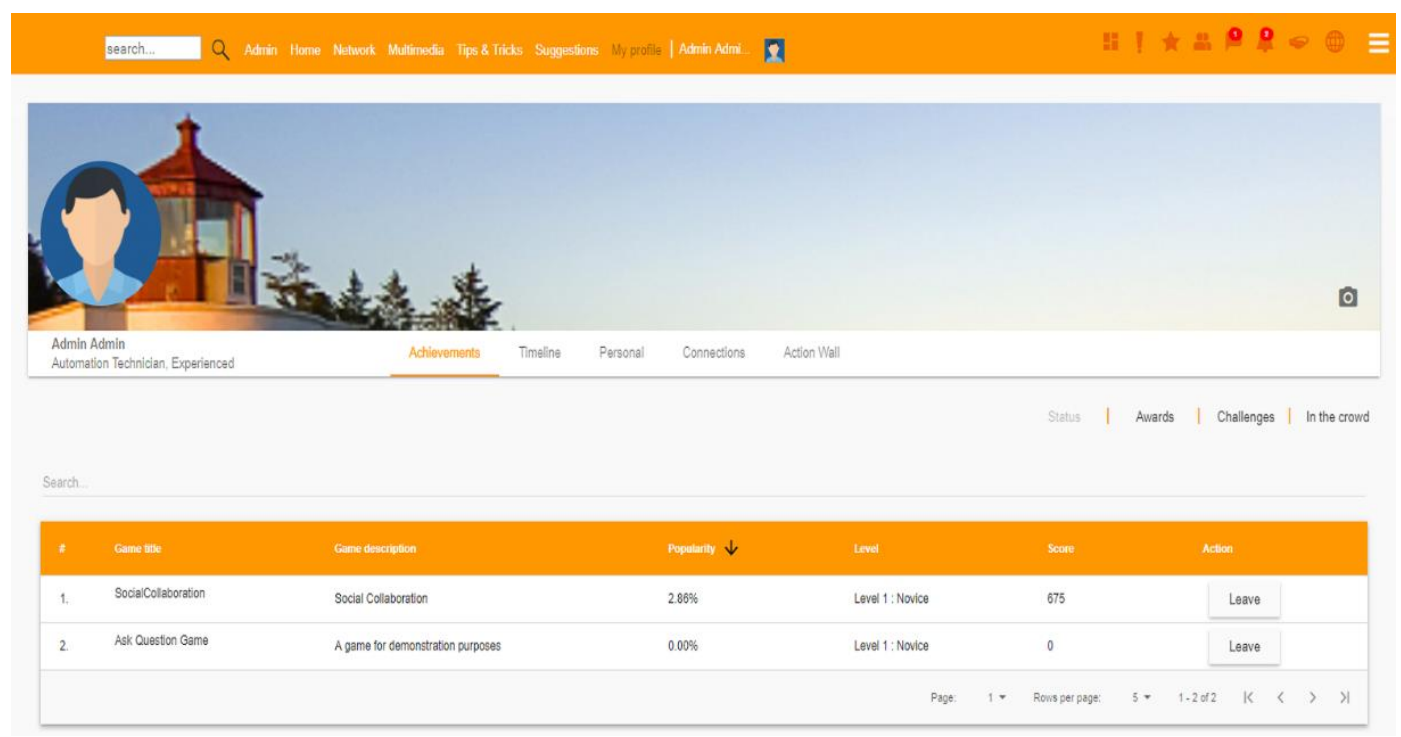

Figure 7. Table of games and progress shown in the user's interface

\section{User evaluation results}

This section describes the methodology that was followed and presents the results of the user evaluation of the collaboration platform that took place at two different industries during pilot testing. Both industries involve a shop floor where assembly lines exist. However, in the first industry (A) more manual operations are required compared to the second industry $(\mathrm{B})$ where production is more automated. 


\subsection{Participants and data collection}

The evaluation process involved three workshops and two data collection periods (DCs) for each industry. The first data collection occurred right after the end of the initial deployment phase, while the second data collection occurred after the deployment of the final version and completion of the demonstrators.

Regarding the workshops, the goal was to inform the involved personnel about the data collection process and to demonstrate the features and the usage of the collaboration platform. The workshops were performed via Internet as webinars. A hands on demonstration of the deployed collaboration platform software was performed in each data collection session. Participants, which were workers and supervisors, provided their feedback separately using questionnaires. Supervisors are the decision makers, therefore, they could use the platform with administrator privileges. Each questionnaire was divided into two sections. The first section included 10 general evaluation questions (System Usability Scale questionnaire) and the second section contained 10 specific evaluation questions according to the following five criteria: Usability, Knowledge integration, Working experience, User acceptance and Overall impact. Participants responding to the questionnaires could choose from five available response options; from 'Strongly agree' to 'Strongly disagree' (Strongly agree, Agree, Neither agree nor disagree, Disagree, Strongly disagree).

System Usability Scale (SUS) analysis allows for determination of usability of a software solution, interpreting answers given to questions related to satisfaction of the user, effectiveness of the system, and efficiency. SUS is a user centered evaluation method and returns relevant and dependable results even for small samples. The SUS score range is from 0 to 100 (the higher the better), where a value over 70 is considered acceptable, while a value over 80 is excellent. An extensive study regarding interpretation of the SUS score can be found in [28].

For the results analysis the five possible responses in each specific evaluation question were grouped into 3 categories. The responses 'Strongly agree' and 'Agree' were grouped to 'Positive' category and the responses 'Disagree' and 'Strongly disagree' were grouped to 'Negative' category. Therefore, a 100\% 'Positive' score does not imply that the participants were fully satisfied.

Demographics information of the participants, such as age and experience level, was also collected at each workshop and are included for reference. Data collection was based on two questionnaires, one on Gamification and one on Collaboration, for both workers and supervisors. It should be noted that the use of collaboration platform without the gamification features could not be evaluated as gamification was a feature of the platform since the beginning.

\subsection{Evaluation criteria}

The following evaluation criteria, which are described next, were considered in this study, in order to capture various aspects of the effects of the system on the users. Related statements that are included in the second section of the questionnaire that was provided to the workers, are presented in Table 2.

Usability: It is the degree to which a software can be used by end users to achieve objectives with effectiveness, efficiency, and satisfaction in a quantified context of use [29]. The usability criterion incorporates efficient visualization, simple navigation and control, focusing on the user work experience.

Knowledge integration: Even though the term 'knowledge integration' is used in different ways in the literature, it can be defined as the exchange of information and knowledge among the members of a community and the creation of the common basis of knowledge or understanding [30]. The use of this criterion in the present study refers to the 
pag. 98

evaluation of the efficiency of the knowledge collected through the collaboration platform, in terms of management, exploitation, quality and completeness.

Working experience: The perception of the user's working experience is about motivations, attitudes, expectations, behavioral patterns and constraints. The introduction of the gamification-enabled collaboration platform affects the work environment and the perception of working experience. The objective is to receive feedback from workers and supervisors on several aspects related to their work experience such as work organization and quality of assistance.

User acceptance: It is often the pivotal factor determining the success or failure of an information system project [31]. In the context of this study, it refers to the willingness of the users (both workers and supervisors) to use the proposed solution. A high rating in the user acceptance criterion means that the solution is useful for the user and works as expected.

Overall impact: This is a general purpose criterion which is used for assessing the overall added value that the platform offers to the end users, including the impact on daily activities, job satisfaction and well-being of the workers. Furthermore, from the business facilitation point of view, cost of installation and maintenance is also of interest.

Table 2. Indicative statements included in the questionnaire that was provided to the workers

\begin{tabular}{|c|c|}
\hline Criterion & Statement \\
\hline Usability & $\begin{array}{l}\text { I feel more motivated and playful in my workplace due to the usage of the } \\
\text { platform }\end{array}$ \\
\hline Knowledge integration & I have submitted several suggestions using the platform \\
\hline Working experience & The platform contributes to the reduction of work-related stress \\
\hline User acceptance & I am motivated and engaged to use the platform \\
\hline User acceptance & The platform improves the sense of team spirit \\
\hline User acceptance & I think that gamification tools increase the attractiveness of the shop floor \\
\hline User acceptance & $\begin{array}{l}\text { The platform helps me feel as a member of a community that uses novel } \\
\text { ways for social interaction and communication }\end{array}$ \\
\hline Overall impact & The features and the functionality of the platform are satisfying \\
\hline Overall impact & The platform is applicable at the shop floor \\
\hline Overall impact & The interface is aesthetically attractive \\
\hline
\end{tabular}

\subsection{Industry A results}

In industry A, 11 workers participated in the first data collection period, while 16 workers participated in the second data collection period. The distribution of the participants with regard to experience level and age is shown in Figure 8. It can be observed that workers of various experience level participated with the majority being up to 39 years old. The categorization of responses to Positive, Neutral, and Negative at each data collection session and per each criterion studied (Usability, Knowledge integration, Working experience, User acceptance, Overall impact), is depicted in Figure 9. 

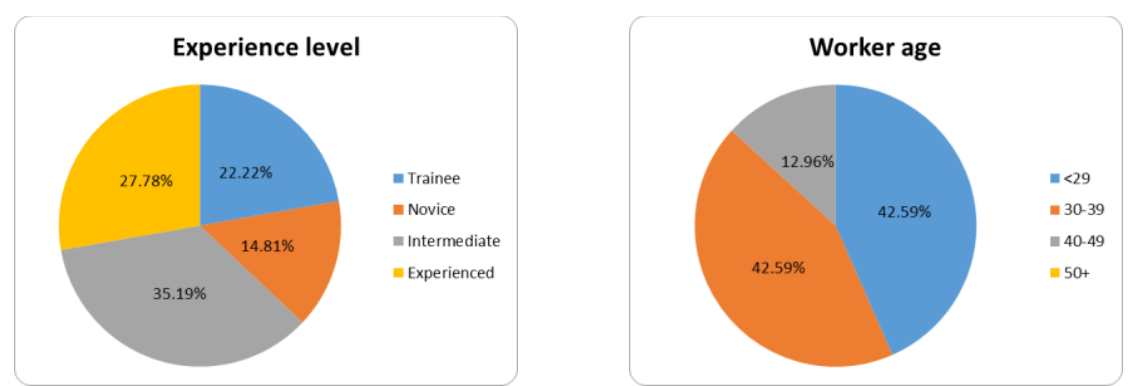

Figure 8. Workers' experience level and age distribution in industry A

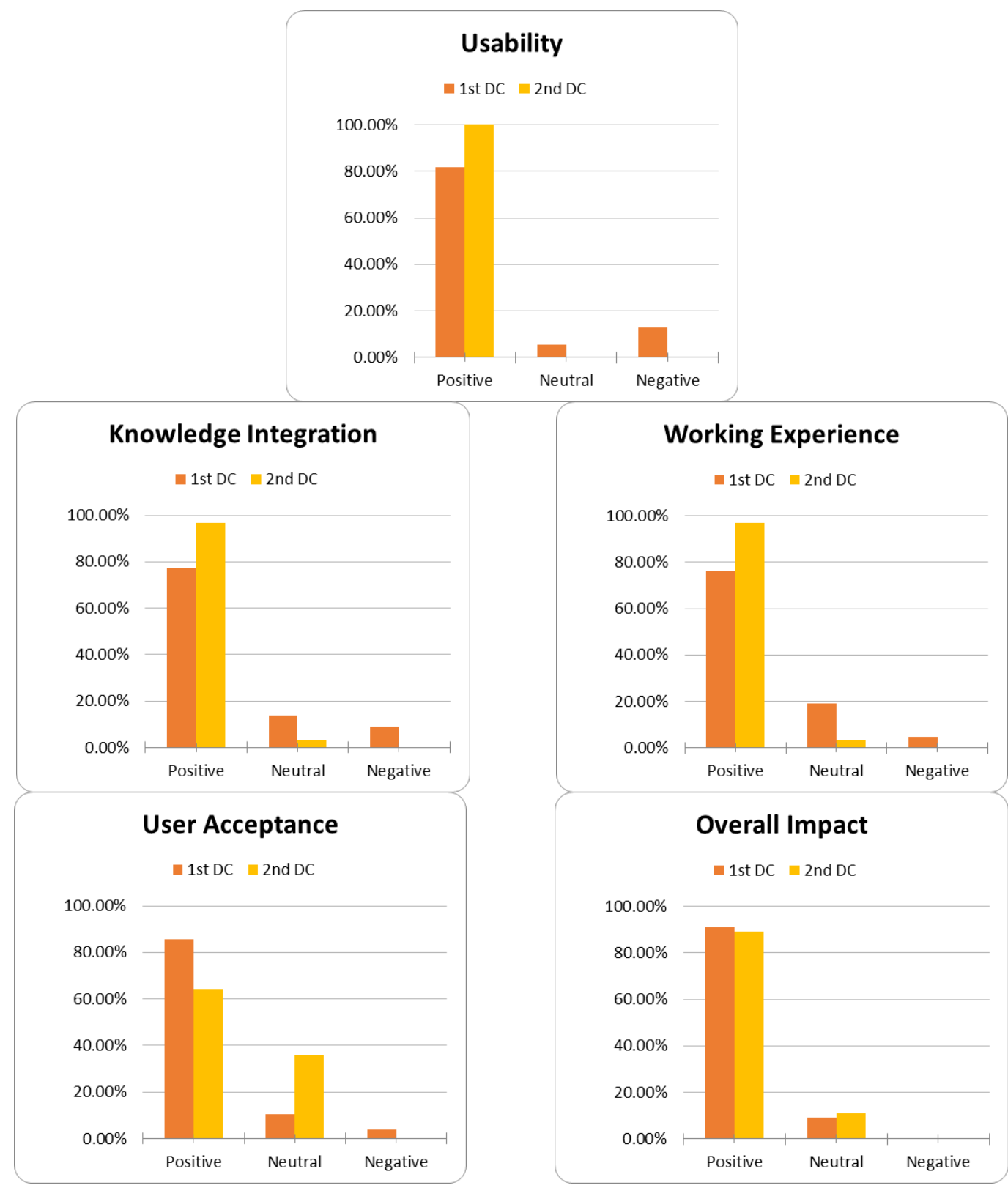

Figure 9. Workers' feedback: (a) Usability, (b) Knowledge integration, (c) Working experience, (d) User acceptance, (e) Overall impact

Regarding the participation of supervisors in the evaluation, 5 supervisors participated in the first data collection period, while 7 supervisors were employed in the second data collection period. The distribution of the participants with regard to experience level and 
pag. 100

age is shown in Figure 10. The majority of supervisors had high experience level and aged between 40 and 49 years old. The categorization of responses to Positive, Neutral, and Negative at each data collection session and per each criterion studied, is depicted in Figure 11. SUS questionnaire scores in the first and the second DC are presented in Table 3. It can be noted that in all cases, scores are above 80 .
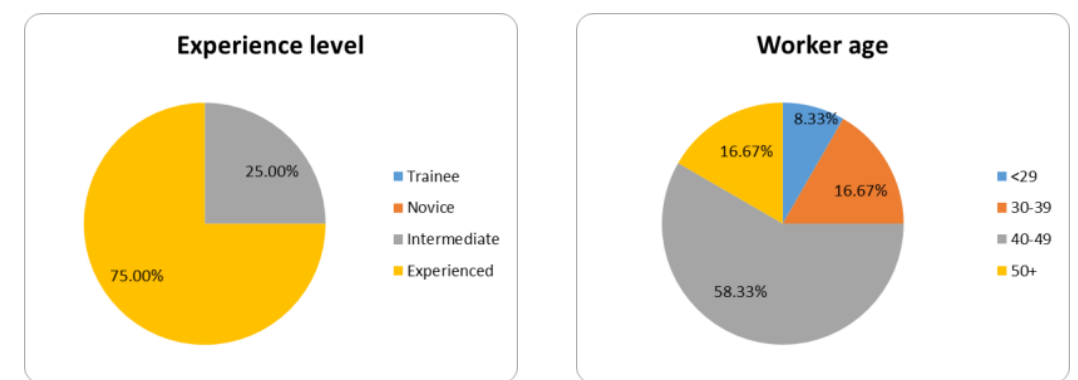

Figure 10. Supervisors' experience level and age distribution in industry A

Table 3. SUS scores in industry A

\begin{tabular}{c|c|c}
\hline User Group & $\mathbf{1}^{\text {st }}$ Data Collection & $\mathbf{2}^{\text {nd }}$ Data Collection \\
\hline Workers & 80.7 & 84.8 \\
\hline Supervisors & 90.5 & 87.5 \\
\hline
\end{tabular}

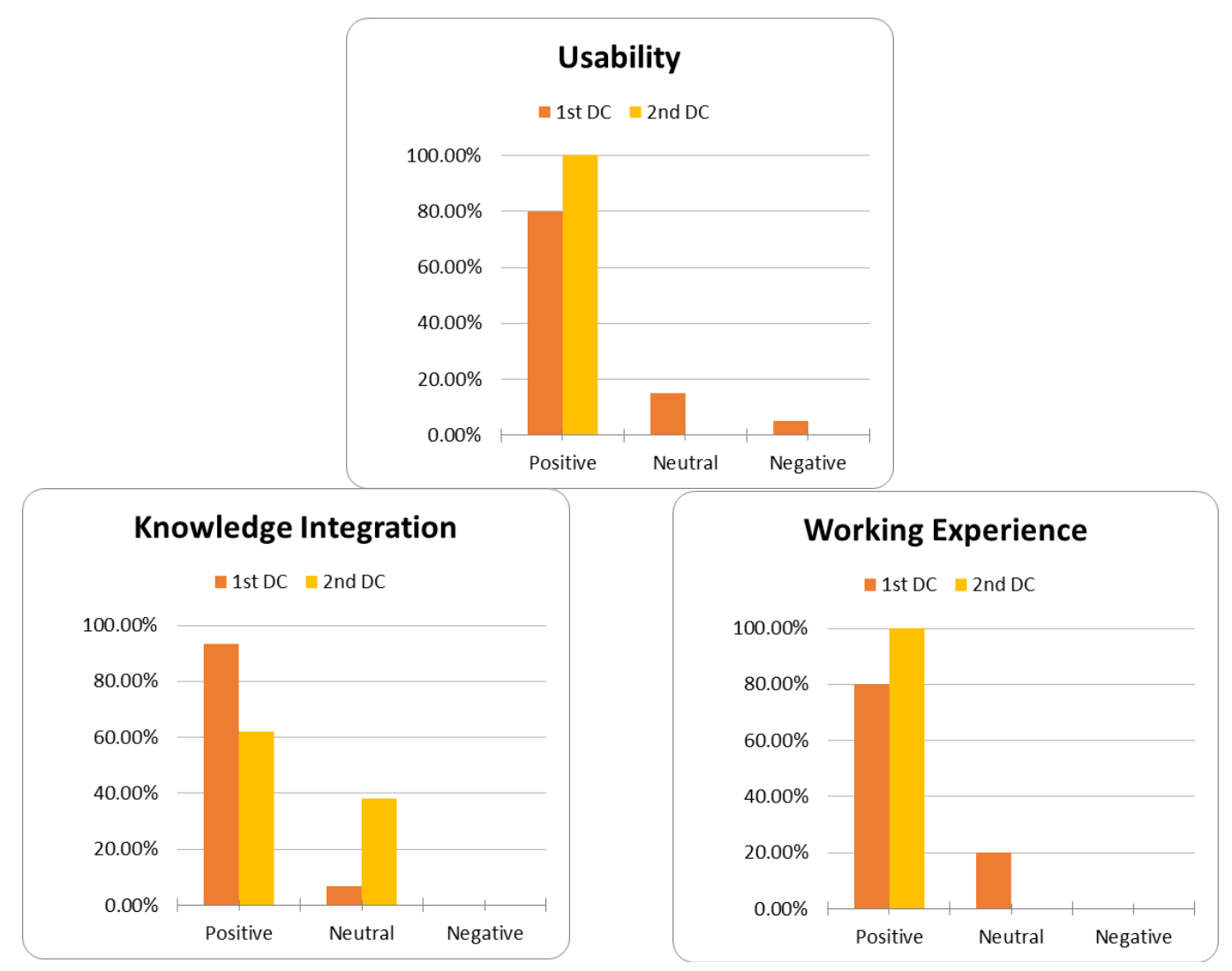



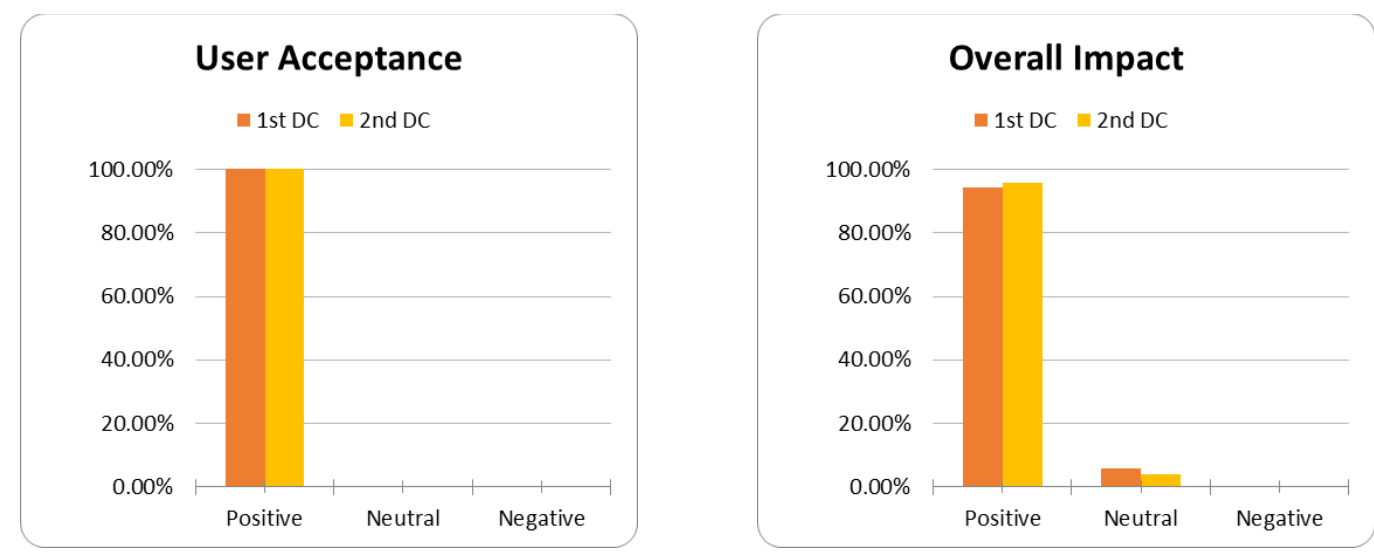

Figure 11. Supervisors' results: (a) Usability, (b) Knowledge integration, (c) Working experience, (d) User acceptance, (e) Overall impact

\subsection{Industry $B$ results}

In industry B, 6 workers participated in the first data collection period, while 20 workers participated in the second data collection period. The distribution of the participants with regard to experience level and age is shown in Figure 12. It is worth noting that about $60 \%$ of workers had at least intermediate experience level, with the majority being up to 39 years old. The categorization of responses to Positive, Neural and Negative at each data collection session and per each criterion studied, is depicted in Figure 13.
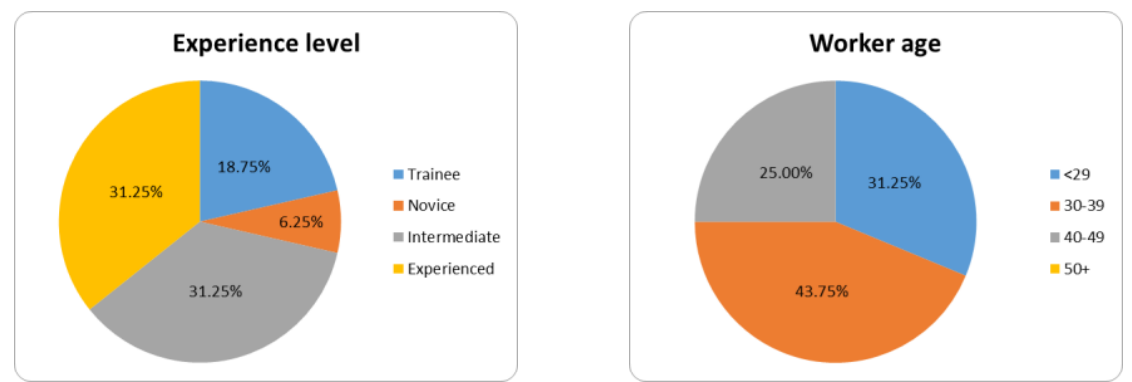

Figure 12. Workers' experience level and age distribution in industry $B$

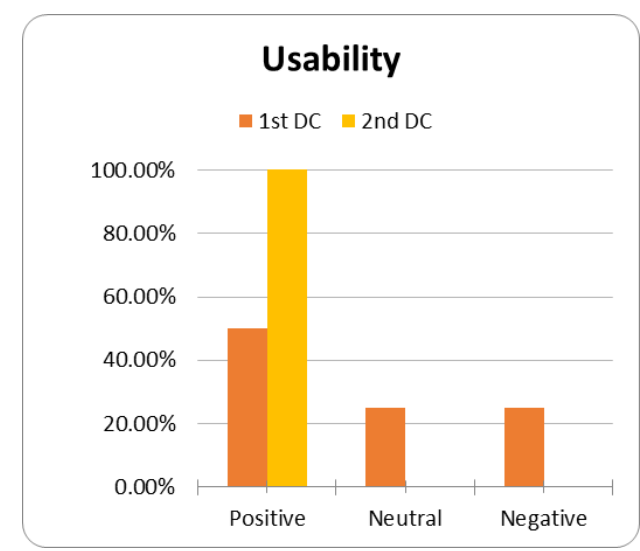



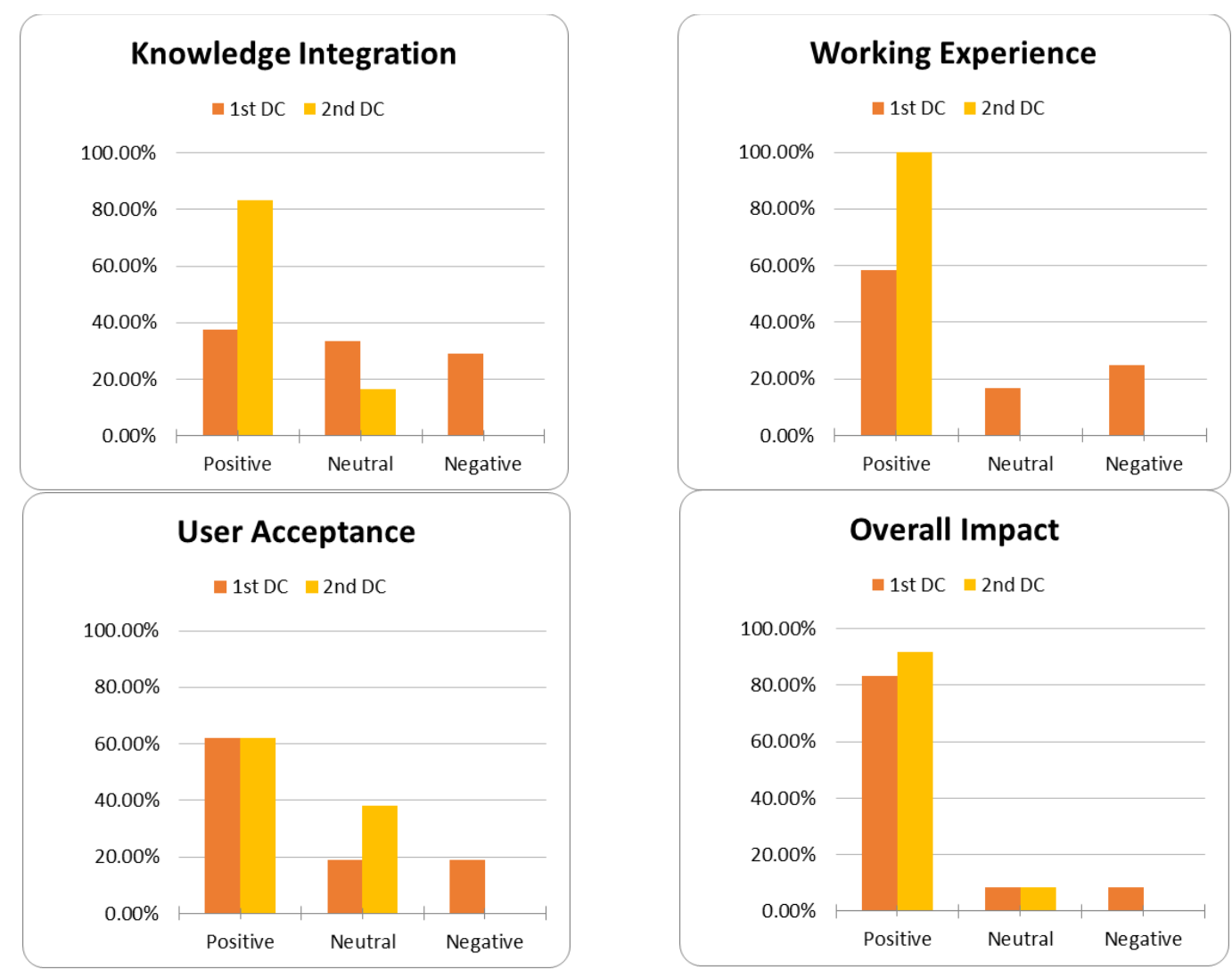

Figure 13. Workers' feedback: (a) Usability, (b) Knowledge integration, (c) Working experience, (d) User acceptance, (e) Overall impact

Questionaires were delivered to supervisors in this factory as well. 6 supervisors participated in the first data collection period, while in the second data collection period, 20 supervisors were employed. The distribution of participants with regard to experience level and age is shown in Figure 14. Supervisors of various experience level participated, with most of them having intermediate experience. In addition, over $90 \%$ were up to 39 years old. The categorization of responses to Positive, Neutral, and Negative at each data collection session and per each criterion studied, is depicted in Figure 15. SUS questionnaire scores in the first and the second DC are presented in Table 4. Even though SUS scores were not too high in the first DC, considerable improvement was observed in the second DC.
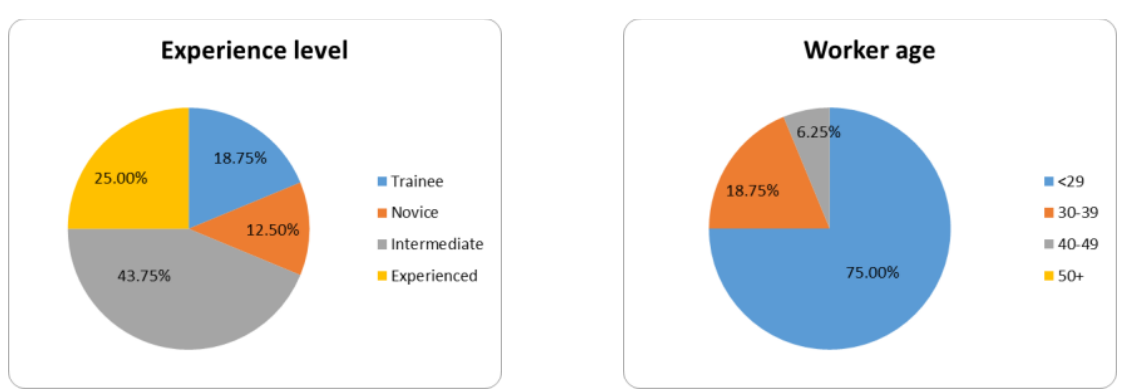

Figure 14. Supervisors' age and experience level distribution in industry $B$ 


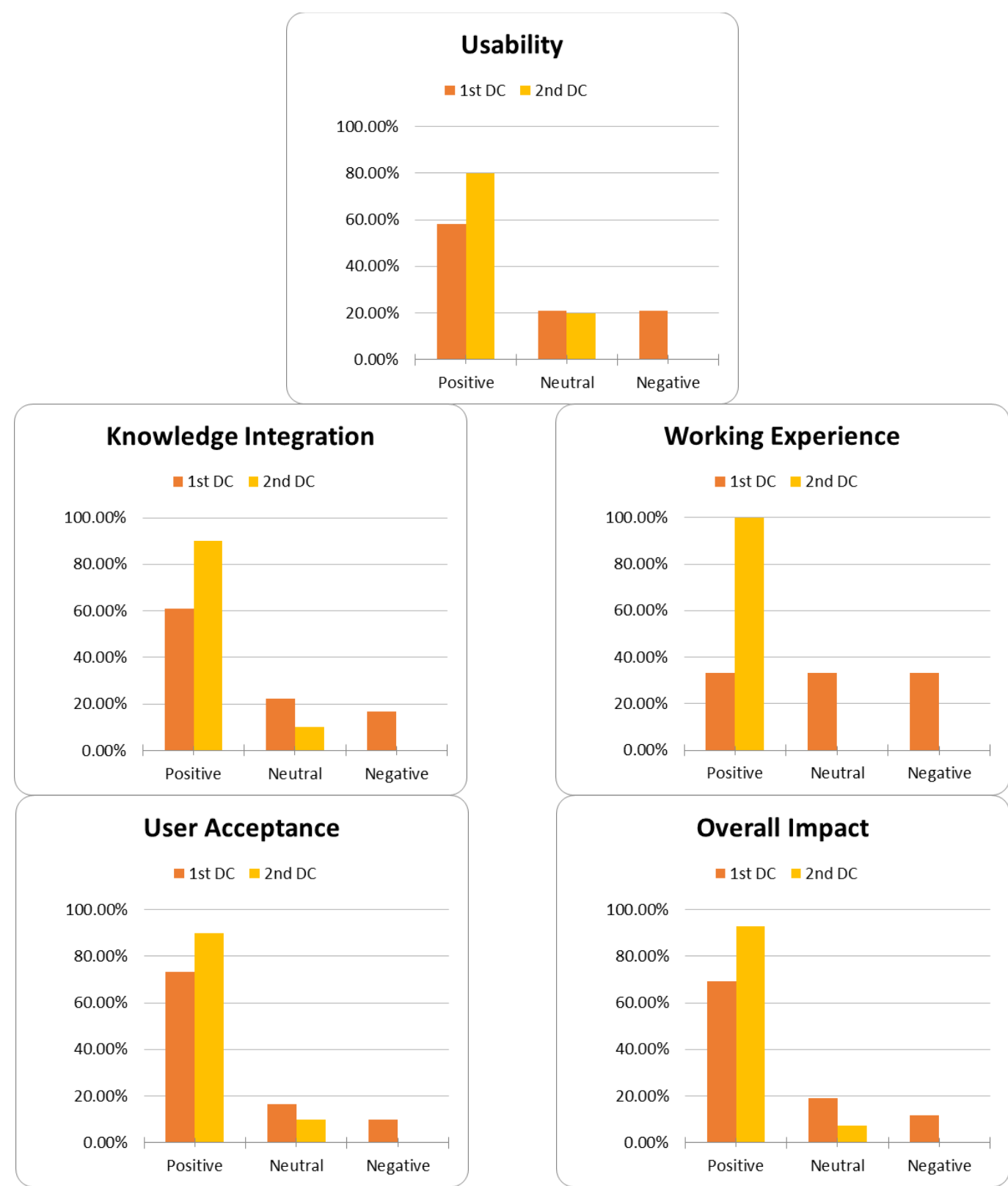

Figure 15. Supervisors' results: (a) Usability, (b) Knowledge integration, (c) Working experience, (d) User acceptance, (e) Overall impact

Table 4. SUS scores in industry $B$

\begin{tabular}{c|c|c}
\hline User Group & $\mathbf{1}^{\text {st }}$ Data Collection & 2 $^{\text {nd }}$ Data Collection \\
\hline Workers & 69 & 81 \\
\hline Supervisors & 70.4 & 80 \\
\hline
\end{tabular}

\subsection{Discussion}

In this section, we discuss the results and other topics that are proposed to be taken into account when gamification is applied to work context. Generally, positive evaluations were received from both industries, especially for 'Working experience' and 'Overall impact' criteria. In some cases, there were contradictory results on a given criterion between workers and supervisors of the same industry, but this can be explained by their different 
roles and functionalities of the platform. Furthermore, in almost all cases, positive ratings were increased in the second data collection period, which indicates that the corrective actions that were made based on user feedback were successful. It is worthwhile to note that no 'Negative' ratings were documented during the second data collection period. Results per industry are analyzed in further detail in the next two paragraphs.

In industry A, even though the workers were very positive about usability, knowledge integration and working experience, especially in the second data collection period, about $40 \%$ 'Neutral' responses were documented on user acceptance criterion. Moreover, the evaluations received from the supervisors were totally positive in all criteria except Knowledge integration. In the second data collection period, about $38 \%$ of answers were rated as 'Neutral'. This can be due to many reasons. Perhaps the quality of knowledge collected was not high, or some supervisors expected higher volume of information to be present in the database. SUS scores were very good in the two DCs. For workers, there was an increase of about 4 units in the second DC. On the contrary, in the evaluation fulfilled by supervisors, SUS slightly decreased by 3 units in the second DC, from 90.5 to 87.5.

In industry $\mathrm{B}$, as far as the evaluation from workers is concerned, Knowledge integration and User acceptance criteria received 'Neutral' ratings, of about $20 \%$ and $40 \%$ respectively. On the contrary, it seems that supervisors were very satisfied, as the positive ratings were at least $80 \%$ in all criteria. However, the score on usability was lower compared with the one documented in industry A. SUS scores of industry B in the first DC were 69 and 70.4, derived from workers and supervisors, respectively. In the second DC, SUS scores were improved significantly to 81 (workers) and 80 (supervisors). By comparing the evaluation results between the two different industries, we conclude that there is no major deviation, even though SUS scores of industry B were somewhat lower than the ones of industry A.

Based on feedback received, useful conclusions that can be used as guidelines when developing a similar system were drawn. The gamified social collaboration platform has been designed by taking into account special requirements of the industrial working environments, such as the use of simple visualizations and gamification elements that do not distract the worker. This approach seems to have contributed to the highly positive user ratings, as the evaluation revealed. Regarding the knowledge integration criterion, such collaboration platforms have to include mechanisms for evaluation and control of the content that is shared by the workers, in order to keep only information of high quality. Proper design of the user interface was also proved to be important. Initially, the process that should be followed in order to create a new game was not clear to the administrator. Therefore, the user interface was improved so it can guide the user to the required steps in order to create gamification elements. In addition, tips are shown to the administrator while he/she is hovering over items using the mouse. Flexibility is an important characteristic that a gamification engine should have. For example, the gamification engine evaluated in this work, allows modification of games according to feedback. The addition of new awards to an existing game which is in progress, can increase motivation and users' interest.

Involvement from managers and supervisors during the development process was very beneficial, especially for the design of gamification functionalities and requirements definition. This is to assure that the system will support the required functionalities and gamification integration will be on par with the company's goals. The extraction of statistics about the user activities from the gamification engine is another feature that can be useful. By logging users' actions and participation level in each gamified activity, game designers and researchers can utilize the information collected in order to analyze how the game influence players' behavior. As a result, game designers can adapt the gamified activities to the needs of specific players, or improve the effectiveness of games in general when collected statistics must be anonymous. 


\section{$5 \quad$ Future work for improving gamification efficiency}

Driven by the evaluation results presented in the previous section, the adoption of adaptive gamification is introduced, as it has the potential to improve gamification efficiency and by extension, the overall user experience and efficiency of the collaboration platform. To this end, extensions to the gamification engine are proposed in this section.

The key idea is to take advantage of monitored game progress data and worker-related profile information that is stored in the database in order to improve design of gamified tasks and enhance worker motivation. Modern factories' IT systems provide interfaces for entering personalized information of employees. This information can be then used for progress self-monitoring or task assignment.

The gamification engine could provide recommendations to the administrator based on the status of each gamified task that can be used in order to re-design it towards making it more attractive for the workers. This can be achieved by the engine through the collection and analysis of monitored game progress data. For instance, if there is decreasing interest on behalf of workers in participating to the gamified task's activities, a recommendation to add extra awards can be generated. Similarly, in case some awards are not earned in a reasonable time period, a recommendation to properly adjust the award's rules should be provided to the administrator.

Another feature that could be implemented concerns the exploitation of information about worker's training needs (based on current skills and experience) along with worker's participation level in a game, in order to enhance worker motivation to watch training videos. To motivate a worker to watch training videos, the gamification engine could automatically create an additional rule to give a badge that is pre-stored in the database, and notify the worker about the required actions in order to unlock this special award.

\section{Conclusions}

In this paper, user acceptance evaluation has been conducted on a gamification-enabled collaboration and knowledge sharing platform that has been developed. The platform, which allows the employees to make discussions, provide suggestions, and share multimedia files related to work, has been evaluated through questionnaires at two manufacturing industries. Evaluation was made in terms of five different criteria, namely, usability, knowledge integration, working experience, user acceptance and overall impact. Participants evaluated the platform twice, right after two separate data collection periods.

Results showed that the ratings received from both workers and supervisors were very positive, especially for working experience and overall impact. In almost all cases, user ratings were improved considerably in the second data collection period, which indicates that user feedback after the first data collection was incorporated successfully. This can be confirmed by the fact that no negative ratings were documented during the second data collection period. By comparing the evaluation results between the two different industries, we conclude that there is no major deviation. SUS scores of industry B were somewhat lower than the ones of industry A, however this could be due to different expectations and experience of the participants.

We also highlighted important aspects that should be taken into account when developing similar platforms for industrial environments, and provided guidelines based on user feedback. Furthermore, as there is still room for improvement, the adoption of adaptive gamification could help to improve gamification efficiency, and by extension, the overall value of the collaboration platform in an enterprise. Concepts for improving gamification efficiency of the platform that take into account additional information about the users, 
were presented. In particular, rule-based adaptive gamification can be applied to increase motivation of particular users.

One limitation of the study is that it was not possible to evaluate the platform when gamification is not employed, due to the fact that the platform was designed to support gamification since the beginning. Furthermore, the number of participants in the evaluation was not so extensive, due to limited availability. However, the findings of this work provide insights into the implementation of highly configurable serious games in industrial work environments.

\section{Acknowledgement}

This project has received funding from the European Union's Horizon 2020 research and innovation programme under grant agreement No 723277 (project Factory2Fit), and has also been partially supported by the European Commission through the project Horizon 2020-Innovation Actions (IA)-636302-SatisFactory. This paper reflects only the authors' view and the Commission is not responsible for any use that may be made of the information it contains.

\section{References}

[1] P. W. Cardon, Community, Culture, and Affordances in Social Collaboration and Communication, International Journal of Business Communication, Vol. 53, Nr. 2, April 2016. https://doi.org/10.1177/2329488416635892

[2] T. G. Cummings, C. G. Worley, "Organisation development and change", Thomson SouthWestern, 2004.

[3] S. Deterding, S. L. Björk, L. E. Nacke, D. Dixon, \& E. Lawley, "Designing gamification: creating gameful and playful experiences". In Proceedings of CHI'13 extended abstracts on Human Factors in Computing Systems, ACM, April 2013. https://doi.org/10.1145/2468356.2479662

[4] D. Djaouti, J. Alvarez, J. P. Jessel, "Classifying serious games: the G/P/S model”, In P. Felicia, (Eds.), Handbook of Research on Improving Learning and Motivation through Educational Games: Multidisciplinary Approaches, IGI Global, 2011. 10.4018/978-1-60960-495-0.ch006

[5] R. Poston, K. Sajja, \& A. Calvert, "Managing user acceptance testing of business applications". In Proceedings of the International Conference on Human-Computer Interaction in Business, Springer, Cham, June 2014.

[6] J. Hamari, J. Koivisto, \& H. Sarsa, "Does gamification work?--a literature review of empirical studies on gamification". In Proceedings of the 47th Hawaii International Conference on System Sciences, IEEE, January 2014. https://doi.org/10.1109/hicss.2014.377

[7] C. Cheong, F. Cheong, \& J. Filippou, "Quick quiz: A gamified approach for enhancing learning". In Proceedings of the Pacific Asia Conference on Information Systems (PACIS), June 2013.

[8] P. Denny, "The effect of virtual achievements on student engagement". In Proceedings of the SIGCHI conference on human factors in computing systems, ACM, April 2013. https://doi.org/10.1145/2470654.2470763

[9] L. Herout, "Application of gamification and game-based learning in education". In Proceedings of the 8th International Conference on Education and New Learning Technologies, ISBN, 2016. https://doi.org/10.21125/edulearn.2016.1212

[10] A. Anderson, D. Huttenlocher, J. Kleinberg, \& J. Leskovec, "Steering user behavior with badges". In Proceedings of the 22nd International Conference on World Wide Web, ACM, May 2013. https://doi.org/10.1145/2488388.2488398

[11] S. Grant, \& B. Betts, "Encouraging user behaviour with achievements: an empirical study". In Proceedings of the 10th Working Conference on Mining Software Repositories, IEEE Press, May 2013. https://doi.org/10.1109/msr.2013.6624007

[12] S. Dale, Gamification: Making Work Fun, or Making Fun of Work?, Business Information Review, Vol. 31, Nr. 2, SAGE, July 2014. https://doi.org/10.1177/0266382114538350 
[13] J. H. Jung, C. Schneider, J. Valacich, Enhancing the Motivational Affordance of Information Systems: The Effects of Real-time Performance Feedback and Goal Setting in Group Collaboration Environments, The International Journal of Management science, Vol. 56, Nr. 4, 2010. https://doi.org/10.1287/mnsc.1090.1129

[14] L. De-Marcos, A. Domínguez, J. Saenz-de-Navarrete, C. Pagés, An Empirical Study Comparing Gamification and Social Networking on E-Learning, Computers \& Education, Vol. 75, Elsevier, June 2014. https://doi.org/10.1016/j.compedu.2014.01.012

[15] P. Petridis, K. Hadjicosta, V. Guang Shi, I. Dunwell, T. Baines, A. Bigdeli, V. Uren, State of the Art in Business Games, The International Journal of Serious Games, Vol. 2, Nr. 1, January 2015. https://doi.org/10.17083/ijsg.v2i1.54

[16] L. Moller, P. K. Hansen, Framing Serious Games Development as a Matter of Business, The International Journal of Serious Games, Vol. 3, Nr. 1, January 2016. http://dx.doi.org/10.17083/ijsg.v3i1.71

[17]D. Kotsopoulos, C. Bardaki, S. Lounis, K. Pramatari, Employee Profiles and Preferences towards Workplace Gamification for Energy Conservation, The International Journal of Serious Games, Vol. 5, Nr. 2, June 2018. https://doi.org/10.17083/ijsg.v5i2.225

[18] J. L. Jurado, A. Fernandez, \& C. A. Collazos, "Applying gamification in the context of knowledge management". In Proceedings of the 15th International Conference on Knowledge Technologies and Data-driven Business (43), ACM, October 2015. https://doi.org/10.1145/2809563.2809606

[19] P. W. Cardon, B. Marshall, The Hype and Reality of Social Media Use for Work Collaboration and Team Communication, The International Journal of Business Communication, Vol. 52, Nr. 3, March 2015. https://doi.org/10.1177/2329488414525446

[20] T. H. Tsai, H. T. Chang, Y. J. Chen, Y. S. Chang, Determinants of User Acceptance of a Specific Social Platform for Older Adults: An Empirical Examination of User Interface Characteristics and Behavioral Intention, PloS one, Vol. 12, Nr. 8, 2017. https://doi.org/10.1371/journal.pone.0180102

[21] O. Korn, P. Muschick, \& A. Schmidt, "Gamification of production? A study on the acceptance of gamified work processes in the automotive industry". In Proceedings of the AHFE 2017 International Conference on Affective and Pleasurable Design, Springer, Cham, 2017. https://doi.org/10.1007/978-3-319-41661-8_42

[22] M. Witt, C. W. Scheiner, \& S. Robra-Bissantz, "Gamification of online idea competitions: insights from an explorative case". In Proceedings of INFORMATIK 2011 GI-Jahrestagung, October 2011.

[23] D. Codish, \& G. Ravid, “Adaptive approach for gamification optimization”. In Proceedings of the 7th International Conference on Utility and Cloud Computing, IEEE, December 2014. https://doi.org/10.1109/ucc.2014.94

[24] M. Böckle, J. Novak, \& M. Bick, "Towards adaptive gamification: A synthesis of current developments". In Proceedings of the 25th European Conference on Information Systems (ECIS), June 2017.

[25] M. Böckle, I. Micheel, M. Bick, \& J. Novak, "A design framework for adaptive gamification applications". In Proceedings of the 51 st Hawaii International Conference on System Sciences, January 2018. https://doi.org/10.24251/hicss.2018.151

[26]É. Lavoué, B. Monterrat, M. Desmarais, S. George, Adaptive Gamification for Learning Environments, IEEE Transactions on Learning Technologies, Vol. 12, Nr. 1, April 2018. https://doi.org/10.1109/tlt.2018.2823710

[27] E. E. Lithoxoidou, S. Doumpoulakis, A. Tsakiris, S. Krinidis, D. Ioannidis, K. Votis, \& D. Tzovaras, "Improvement of the workers' satisfaction and collaborative spirit through gamification”. In I. Kompatsiaris et al. (Eds.), Internet Science, INSCI 2017, Lecture Notes in Computer Science, Springer, Cham, November 2017. https://doi.org/10.1007/978-3-31970284-1_15

[28] A. Bangor, P. Kortum, J. Miller, Determining What Individual SUS Scores Mean: Adding an Adjective Rating Scale, The Journal of Usability Studies, Vol. 4, Nr. 3, May 2009.

[29]E. Din, 9241-11. Ergonomic requirements for office work with visual display terminals (VDTs)-Part 11: Guidance on usability. International Organization for Standardization, 1998.

[30] J. Godemann, Knowledge Integration: A Key Challenge for Transdisciplinary Cooperation, Environmental Education Research, Vol. 14, Nr. 6, December 2008. https://doi.org/10.1080/13504620802469188 
pag. 108

[31]F. D. Davis, User Acceptance of Information Technology: System Characteristics, User Perceptions and Behavioral Impacts, The International Journal of Man-machine Studies, Vol. 38, Nr. 3, March 1993. https://doi.org/10.1006/imms.1993.1022 